\title{
Quantification of Adulterations in Sesame Oil with Inferior Edible Oils by using ATR-FTIR Coupled to Chemometrics
}

\author{
H. Rizki ${ }^{1,2}$, W. Terouzi ${ }^{1}$, F. Kzaiber ${ }^{1}$, H. Hanine ${ }^{2}$, A. Oussama ${ }^{1}$. \\ ${ }^{1}$ Laboratory of Spectro- Chemometrics and environment, Faculty of Science and Technology of Beni Mellal, \\ University of Sultan Moulay slimane, 21000-Beni Mellal, Morocco \\ ${ }^{2}$ Laboratory of development and food safety, Faculty of Science and Technology of Beni Mellal, \\ University of Sultan Moulay slimane, 21000-Beni Mellal, Morocco
}

\begin{abstract}
Recently, sesame oil is highly appreciated by consumers due to its sensory qualities, antioxidants activity and nutritional benefits, despite its high price. Also, the detection and quantification of adulteration both are problem with increasing importance in the consumer's health and food industry. The goal of this study was to use Fourier transform mid-infrared (FT-MIR) spectroscopy coupled with chemometrics for the quantification of the adulteration of sesame oil. Adulteration with sunflower oil, soybean oil or colza oil is one of the most difficult to detect due to the similar composition of them and sesame oil. Adulterations of sesame oil with different percentages of sunflower oil, soybean oil and colza oil were measured using ATR-FTIR spectroscopy. Spectral and reference data were firstly analyzed by principal component analysis. Partial least square regression (PLSR) was used to establish calibration models. Excellent correlations between ATR-FTIR analysis and studied edible oils concentration was obtained for sunflower oil $\left(R^{2} v=0.997\right)$, soybean oil $\left(R^{2} v=\right.$ 0.993) and colza oil $\left(R^{2} v=0.994\right)$. PLS models demonstrated the feasibility of quantifiing successfully adulterations of sesame oil in the 0-35\% weight ratio range of edible oils with a rapid and inexpensive tool without the need for sample preparation.
\end{abstract}

Keywords : ATR-FTIR spectroscopy, Adulteration, Edible oils, Sesame oil, Partial least squares regression.

\section{Introduction}

Sesame seeds are important oil seed crop cultivated in Asia and Africa for a long time. Recently, sesame oil has received great attention because of its bioactive compound including tocopherols, phytosterols, resveratrol, flavonoids [1], and also its sensory qualities and antioxidants activity. The compounds of sesame oil have physiological functions, such as estrogenic activity, providing anti-inflammatory functions, decreasing blood lipids and arachidonic acid levels [2], [3]. Sesame oil has a long self life and is used as cooking oil or seasoning and margarines, because of its high stability and resistance to oxidative deterioration. This resistance is due essentially to the high amount of lignin compounds (1034 ppm) such as sesamol, sesaminol and $\alpha$ tocopherol [4]. Sesame oil is known also by containing nearly $85 \%$ unsaturated fatty acids specially high contents of essential linoleic and linolenic acids which make a good food source. Sesame oil is manufactured by solvent extraction from raw sesame seeds followed by the refining process, or by pressing sesame seeds without refining [5].

In fact, Sesame oil has attracted the attention of public due to its beneficial constituents to the health. It has a distinctive flavor and possesses higher oxidative stability than other vegetable oils. But except in a few countries of Asia, the production of sesame oil is still suffering the constraints on its valuation and profitability. Also, sesame oils can be considered expensive compared to other vegetable oils, which makes its marketing a bit difficult in some countries. That, making it prone to adulteration with less expensive oils (other vegetable oils with low price). Vegetable oils such as rapeseed oil, soybean oil and sunflower oil are used as an adulterant for high-cost oil like olive oil and argan oil.

Adulteration is a major issue in the high quality oils market. The adulteration of food products presents a huge importance for consumers, food processors, regulatory agencies and industries, done for economic reasons. It involves replacing or dilution of high cost ingredients with lower and cheaper ones [6], [7]. Thus, make it a serious problem for oil suppliers and could be a threat for consumers health. Then, it become very important of food product to identify if as being adulterated and with what substance. Also, it needs to aply a rapid quantitative analysis of the adulterant.

Detection of adulteration is important for the protection of wealth and health of consumers. several methods have been used to detect the authenticity and adulteration of oil such as gas chromatography(GC) for the quantification of fatty acids, triglycerols, sterols and hydrocarbons [8], and classic wet chemistry [9], and high performance liquid chromatography (HPLC) [10], but those methods are time, destructive and unsuitable for rapid screening. For this reason, research has been done to find a non-destructive solution, rapid, easy and efficient for the detection and quantification of oil adulteration. 
Many studies have been carried out on the detection and quantification of olive oil adulteration with vegetable oils [11], [12], [13], [14]. Also, some publications covering argan oil adulteration are available in literature. Rezanka \& Rezankova (1999) [15] and Hilali \& al. (2007) [16] used gas chromatography analysis and applied statistical methods to detect adulterations of argan oil. In a continuation of this research, Rachid Salghi \& al. (2014) [17], applied high-performance liquid chromatography-evaporative light scattering detection. Gonzálvez \& al. (2010) [18], arrived to detect adulteration by inductively coupled plasma optical emission spectrometry. However, only few publications and informations are available on sesame oil adulteration. An "electronic nose" has been used by Hai \& Wang (2006) [19]. Peng \& al. (2015) [20] used gas chromatography (GC) and multivariate data analysis for detection and quantification of adulteration of sesame oil with vegetable oils. Lee \& al. (2013) [21] used a simple, modified colorimetric Villavecchia test and triacylglycerol (TAG) profile analysis to assess the authenticity of sesame oil. A simple and rapid detection technology was proposed by Zhang \& al. (2016) [22] based on ion mobility spectrometry (IMS) fingerprints to determine potential adulteration of sesame oil.

Today, the application of Fourier transform infrared (FTIR) spectroscopy has increased in food studies, and particularly has become a powerful analytical tool in the study of edible oils and fats [23]. FTIR has been also successfully used to detect olive oil adulteration [24], [25].The latter technique is often coupled with chemometrics methods that can be used to assign the measured spectrum to a category in a presentation, and can be used for monitoring the quality aspects of fats and oils [26].

In this case, the aim of the present work is to develop a new application of the FTIR-PLS association as a rapid, inexpensive and non destructive authenticity measuring tool, useful to determine the adulteration of sesame oil with other edible oils and also to identify and quantify the percentage of the ruining agent in the blend.

\subsection{Sampling}

\section{Materials and Methods}

Sampling is an important step. Indeed, the reliability and robustness of the method adopted repose largely on the choice and number of samples. Forty eight authentic samples of sesame oil (SO) extracted by soxhlet with hexane as solvant was personally taken from different sources in Morocco. Colza oil, soybean oil, sunflower oil were used as adulterants and were purchased in a local supermarket. For the adulteration study, oil samples were prepared by mixing sesame oil (SO) with colza oil (CO), soy bean oil (SBO), and sunflower (SFO) in the range of 0-35\%. The samples were stored in the dark at ambient temperature until analysis.

There were 144 samples in total, among which 102 samples were randomly for establishing principal component analysis and partial least square (PLS) models. Other 42 samples were used for testing the models. The PLS models were constructed using 34 calibration samples and 14 prediction samples for binary mixtures SO-SFO, SO-SBO or SO-CO in the adulteration range of 0-35\% weight ratios.

\subsection{ATR-FTIR Analysis of Oil Samples}

A spectrometer Bruker Vector 22 equipped with DTGS detector, Globar (MIR) Source and $\mathrm{KBr}$ Germanium separator, was used to record the FTIR spectra. Analyses were carried out at room temperature. The samples were placed on a single bounce ATR Diamant (Pike). Ninety scans were taken at a resolution of $4 \mathrm{~cm}-1$ and averaged. The background was collected before every sample was measured.

Spectra were scanned in the absorbance mode from 4000 to $600 \mathrm{~cm}^{-1}$ and the data were handled with OPUS logiciel. The software (Opus 4.0 MSD) fitted to the infrared spectrometer Fourier transform used in this study allows the automatic acquisition of the spectra without any form of computer manipulation may impair the quality of results. Two drops of each sample of sesame oil were were placed on an Attenuated Total Reflectance cell provided with a diamond crystal. Between spectra, the ATR plate was cleaned in situ by scrubbing with ethanol solution, enabling to dry the ATR.

\subsection{Data Pre-processing Procedures}

In this study, a series of pre-processing elaborations were tested on the spectral data prior to the multivariate calibration. The Savitzky-Golay [27] and Norris gap [28] algorithms were tested for data derivatisation. Standard normal variate (SNV) and multiple scatter correction (MSC) [29] were also tested. PCA and PLSR were carried out on the spectral data. ATR-FTIR spectra were mean centered to remove or minimise any unwanted baseline contribution. The leave-one-out method was employed for cross-validation. This validation was also called full cross-validation. In this technique, one of the calibration samples is removed. The value for the removed sample was then predicted, and the prediction residual computed. The process was repeated with another sample of the data set, and so on, until every sample had been left out once [30]. 


\subsection{Statistical Treatment of Data}

\subsubsection{Principal Component Analysis (PCA)}

PCA, as a linear projection method is based on variance, transforms the original measurement variables into new uncorrelated variables called principal components [31], [32]. It provides a reduction in data set dimensionality and allows linear combinations of the original independent variables that are used to explain the maximum of data set variance [33].

\subsubsection{Partial least squares regression (PLSR)}

Partial least squares regression (PLSR) [34] is popular and the most commonly used multivariate calibration chemometrics methods. It is able to resolve overlapping spectral responses [35]. It assumes a linear relationship between the measured sample parameters (for example, concentration) and the experimentally measured spectra.

PLSR attempts to maximize the covariance between $X$ and $y$ data blocks as it searches for the factor subspace most congruent to both data blocks. A new matrix of weights (reflecting the covariance structure between the $\mathrm{X}$ and $\mathrm{y}$ ) is calculated and provided rich factor interpretation information [36].

In this study, the collected MIR spectra will be used as the X matrix, and the adulterants concentrations of the different samples will be used as the $\mathrm{Y}$ vector.

\subsubsection{Software}

The pre-treatment procedures and all chemometric models were performed by using the Unscrambler X software version 10.2 from Computer Aided Modelling (CAMO, Trondheim, Norway).

\subsection{Spectral diversity}

\section{Results and Discussion}

In the first step, Fourier transform mid infrared (FT-MIR) spectra of pure sesame oil (SO), colza oil, soy bean oil, and sunflower, were obtained. One spectrum is the average of 90 scans of the same sample of oil on FT-MIR. The average spectra of all considered samples are presented in Fig. 1a.

In the second step, FT-MIR spectra of 102 samples, including 34 samples from each of the three binary mixtures studied, were recorded in triplicate and a mean spectrum was calculated for each sample studied. The resultant mean spectra of three binary mixtures are shown in Fig. $\mathbf{1 b}$.

Fig.1 shows the FT-MIR spectra of the studied oils at frequency region of $4000-600 \mathrm{~cm}^{-1}$. The obtained spectra are dominated by typical bands of holocellulosic materials in the $1200-900 \mathrm{~cm}^{-1}$ region [29]. The assignment of functional groups responsible for MIR absorption at these region is as follows: 1160 ( $-\mathrm{C}-\mathrm{O}$ stretch; $-\mathrm{CH} 2$ bending), 1120 ( $-\mathrm{C}-\mathrm{O}$ stretch), 1098 ( $-\mathrm{C}-\mathrm{O}$ stretch), 1032 ( $-\mathrm{C}-\mathrm{O}$ stretch), 965 (trans- $\mathrm{CH}=\mathrm{CH}-$ bending out of plane) [24], [37]. In the $4000-1200 \mathrm{~cm}^{-1}$ region, All the spectra were dominated by two peaks at 2860 and $2850 \mathrm{~cm}^{-1}$, due to bands arising from asymmetrical and symmetrical stretching vibrations of methylene $(-\mathrm{CH} 2)$ groups. The peak at $3008 \mathrm{~cm}^{-1}$ could be assigned to the functinal group (trans $=\mathrm{C}-\mathrm{H}$ stretch), and strong bands between 1750 and $1650 \mathrm{~cm}^{-1}$ arising from the stretching vibration of the ester carbonyl functional groups of the triglycerides. The band of the aromatic ring stretch of the lignin appears at $1604 \mathrm{~cm}^{-1}$. The bands from 1480 to $1450 \mathrm{~cm}^{-1}$ could be assigned to the bending vibrations of the $-\mathrm{CH} 2$ and $-\mathrm{CH} 3$ aliphatic groups [38], [39].

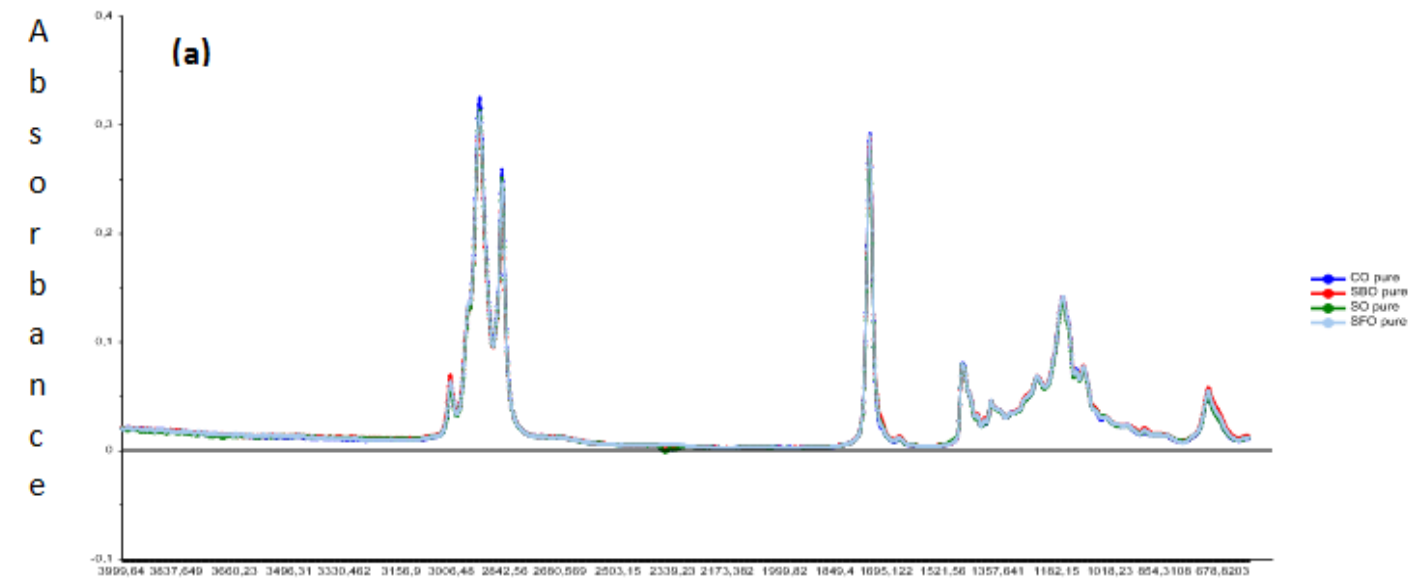

Wavenumbers $\left(\mathrm{cm}^{-1}\right)$ 


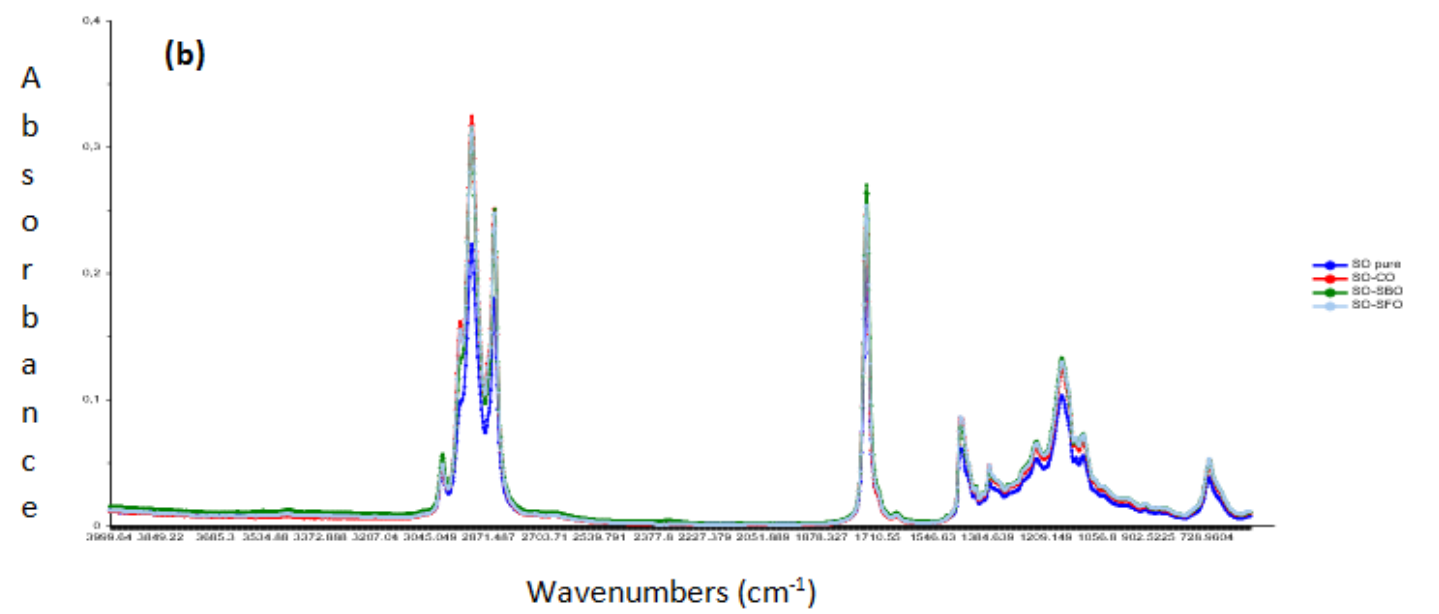

Fig.1. MIR spectra of SO and adulterants at MIR region of 4000-600 cm-1 : (a) colza oil (CO), soy bean oil (SBO), and sunflower (SFO). (b) binary mixtures SO-SFO, SO-SBO and SO-CO

In fact, MIR spectroscopy is a fingerprint technique, allows to differentiate authentic oils and those adulterated with others by observing the spectra changes due to the adulteration [40]. According to Fig.1, the MIR spectra obtained for calibration set of the studied oils to be similar. The detection of adulteration is more difficult, especially when the adulterant has similar chemical composition to that of the original oil [41], [42]. There is an urgent need to control and ensure the quality of sesame oil. Multivariate calibration methods appeared then to be ideal to provide an effective solution, as they allow extracting of unspecific analytical information from the full-spectra or large regions of them.

Since the native FTIR spectra did not furnish enough information to build a reliable prediction model, a preliminary treatment of the data seemed necessary to extract better analytical information. At the same time, a data pre-treatment was considered useful to minimize instrumental problems as baseline fluctuation or noise. Different mathematical elaborations were so explored to handle the spectral data. Derivative elaboration showed the most interesting result. In particular, a significant enrichment in the data variance was reached when the starting data were transformed in derivative signals by means of Gap derivative algorithm. Different mathematical parameters in the derivative procedure were tested and results were optimized when the following parameters were selected: 2nd order, gap size 13.

\subsection{PCA modeling}

Principal component analysis was carried out to detect the presence of any spectral outliers in the spectral data, prior to develop a prediction model using PLS regression.

Many studies indicate that PCA is a useful tool for the identification of spectral outliers in the absorbance spectra of the samples and can be employed to increase the quality of the prediction-model [43]. According to Fig.2 of PCA score plot, the data set contained in total seven spectral « outliers » (number 11 and 49 / Fig.2a ; number 27, 28 and 31 / Fig.2b ; number 15 and 44 / Fig.2c). However, at first, the prediction models (PLSR) were building with all samples including the seven samples to insure their nature (outliers or extreme samples).

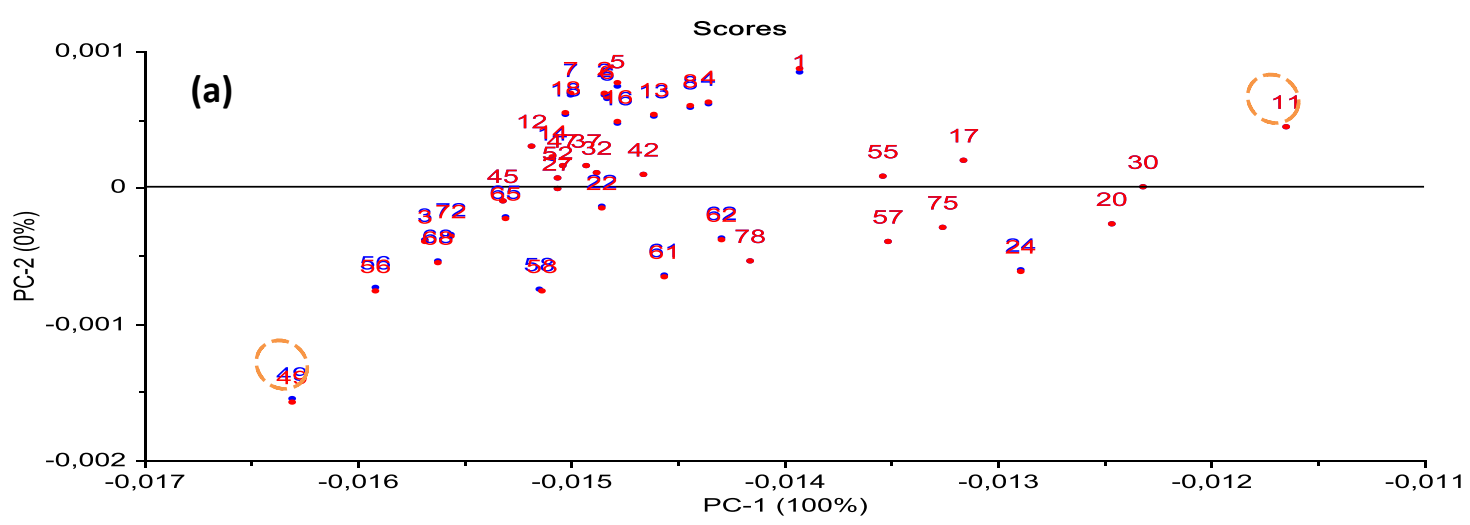



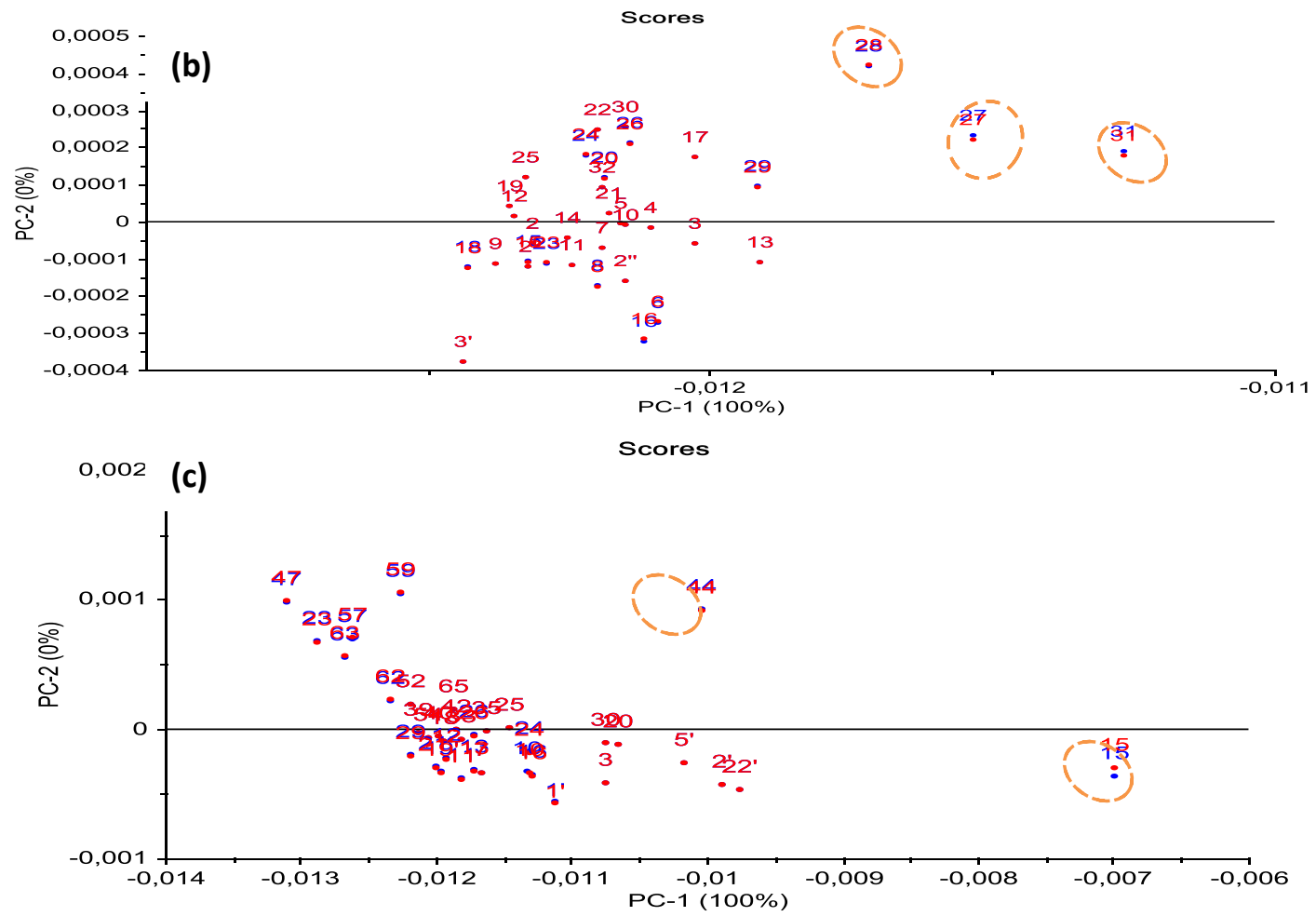

Fig. 2. PC1 / PC2 Score plot by PCA analysis on the calibration set of binary mixtures samples : (a) SO-CO ; (b) SO-SBO ; (c) SO-SFO

\subsection{Partial Least Squares Regression (PLSR) Modeling}

The quantification of colza oil, soybean oil and sunflower oil as adulterants in sesame oil was carried out using PLS algorithm. The PLS model is built by considering the all spectra range $4000-600 \mathrm{~cm}^{-1}$ with $\mathrm{X}$ as variable and the $\mathrm{Y}$ variables is associated to the concentrations of the different adulterants. The data set contained 102 oil samples including the seven spectral « outliers » identified by PCA (Fig.2) because they are considered extreme by PLS. The adulteration of SO with CO, SBO and SFO was individually modelled and three lineal models were proposed. The PLSR models are evaluated using coefficient of determination $\left(R^{2}\right)$ in calibration, root-mean-square error of calibration (RMSEC) and cross validation (RMSECV).
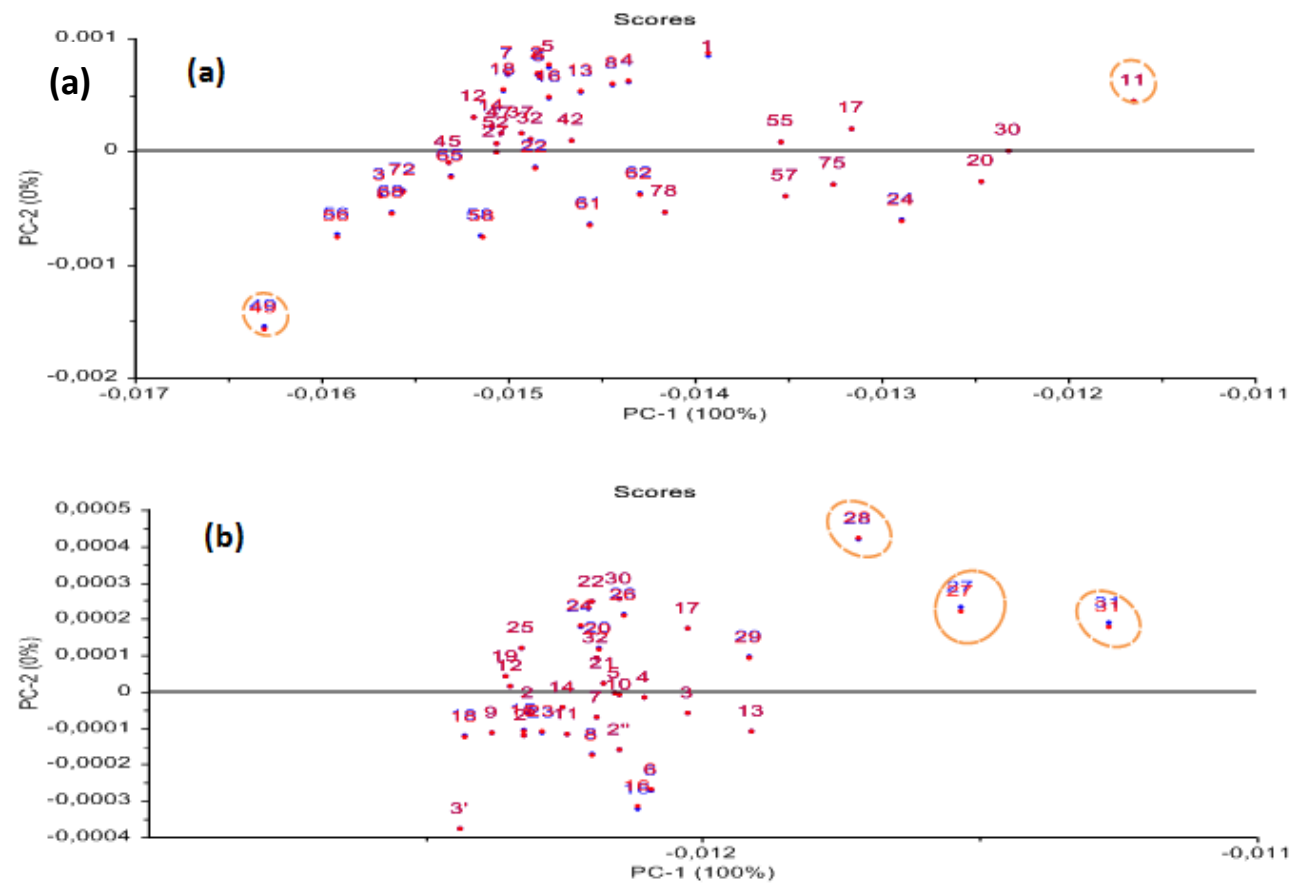


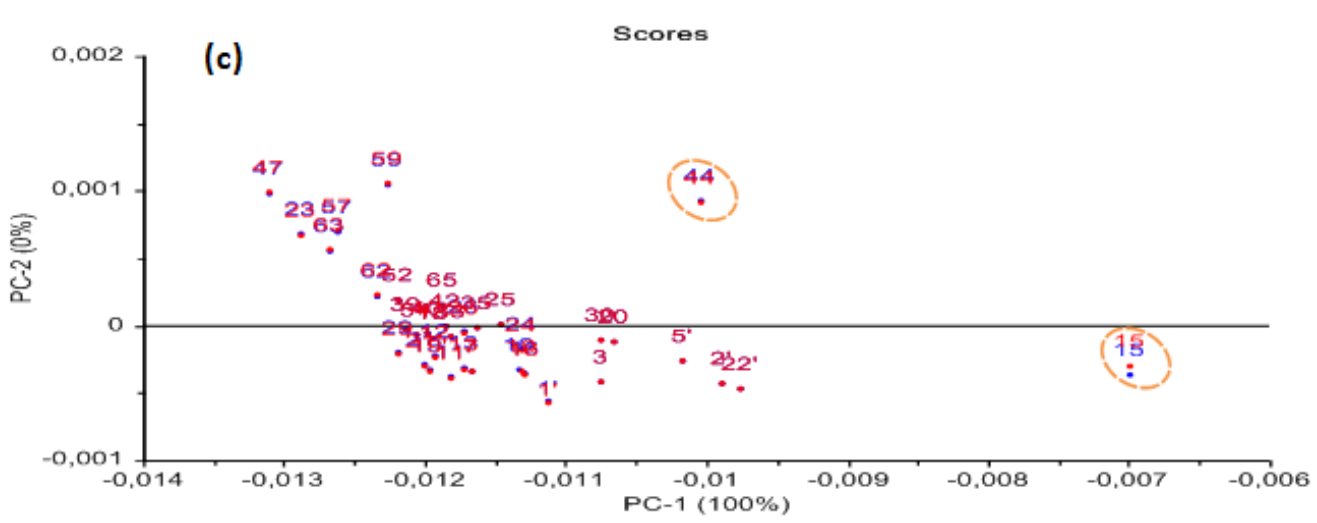

Fig.3. The relationship between actual and estimated concentrations of adulterants in SO, obtained from the final PLS model developed from the FT-MIR spectra : (a) CO in SO. (b) SFO in SO. (c) SBO in SO

Fig.3 shows the PLS models which correlates the « actual» and «predicted » values of CO, SBO and SFO concentrations obtained from FT-MIR spectra. The term «actual» refers to the known concentration of adulterant. The «predicted » refers to a value calculated by the model using spectral data. The difference between the actual and the predicted concentration of adulterants is relatively small with coefficient of determination $\left(\mathrm{R}^{2}\right)$ values above 0.99 for all adulterants. The low value RMSEC $(\leq 1.42)$ indicates the good performance of PLS models [44].

Table1. Explained variances (\%) of LVs used in the PLSR models.

\begin{tabular}{|l|l|l|l|l|l|}
\hline & Explained & Factor 1 (LV1) & Factor 2 (LV2) & Factor 3 (LV3) & Factor 4 (LV4) \\
\hline \multirow{2}{*}{ PLSR (a) } & Calibration & 71.71 & 94.54 & 98.59 & 99.64 \\
\cline { 2 - 6 } & Validation & 70.13 & 93.79 & 98.23 & 99.43 \\
\hline \multirow{2}{*}{ PLSR (b) } & Calibration & 71.15 & 96.11 & 99.51 & - \\
\cline { 2 - 6 } & Validation & 69.39 & 95.44 & 99.40 & - \\
\hline \multirow{2}{*}{ PLSR (c) } & Calibration & 76.34 & 94.25 & 96.50 & 99.87 \\
\cline { 2 - 6 } & Validation & 74.96 & 93.36 & 94.34 & 99.73 \\
\hline
\end{tabular}

(a) CO in SO. (b) SFO in SO. (c) SBO in SO

Validity of the models was checked by running several diagnostics including $\mathrm{R}^{2}$, root mean standard error of calibration (RMSEC) and root mean standard error of cross validation (RMSECV).

Root mean square error of cross-validation (RMSECV), recovery percentage and coefficient of determination $\left(\mathrm{R}^{2}\right)$ were used as parameters to determine appropriate number of latent variables (LV) [45], [46].

The determination of latent variables number was based on the statistical parameters that they offer the highest values of $\mathrm{R}^{2}$ and the lowest values of error, either in calibration or in prediction models. The statistical parameters RMSEC, RMSECV and $\mathrm{R}^{2}$ are summarized in Fig.3. The coefficient of determination $\left(\mathrm{R}^{2}\right)$ of 0.99 , RMSEC lower than 1.42 and RMSECV lower than 1.58, could be considered satisfactory. The four latent variables (factors) were sufficient for describing PLS model for both adulterants CO and SFO, but just three factors for SBO with an explained variances above 99\% (Table1).

\subsection{Determination of Adulterant oils Concentrations in the New Samples (External validation)}

Finally, in order to verify the applicability and how reliable this models in estimating the concentration of every adulterated oil, the external validation process was carried out.

PLS models are used to predict adulterant oil concentrations in new samples. The new samples have the same matrix effects as samples of calibration set. In this step, the models were subdued to validation procedure by quantifying the new objects.

The PLS models were applied to a group of unknown samples from different oils of three adulterants (14 external oil samples of each adulterant), the results are listed in Table2. The most accurate quantification of every adulterating oil is determined using their respective individual models.

The deviations of prediction of adulteration in sesame oil by FT-MIR spectroscopy were between 0.72 and 1.84, which were very satisfied (Table2).

Table2. Prediction results of the extent of adulteration of CO, SBO and SFO in sesame oil by FT-MIR spectroscopy coupled with PLS regression.

\begin{tabular}{|l|l|l|l|l|l|l|l|l|}
\hline SO-CO & \multicolumn{2}{l|}{ SO-SBO } & \multicolumn{2}{l|}{ SO-SFO } \\
\hline Samples & Pred. & Dev. & Samples & Pred. & Dev. & Samples & Pred. & Dev. \\
\hline A1 & 6.2961 & 1.8484 & B1 & 11.5046 & 1.3779 & C1 & 2.7596 & 0.7216 \\
\hline A2 & 6.5626 & 1.3557 & B2 & 9.7304 & 1.3255 & C2 & 4.3017 & 0.8057 \\
\hline
\end{tabular}


Quantification of Adulterations in Sesame Oil with Inferior Edible Oils by using ATR-FTIR Coupled...

\begin{tabular}{|l|l|l|l|l|l|l|l|l|}
\hline $\mathbf{A 3}$ & 4.7253 & 1.2376 & $\mathbf{B 3}$ & 7.7021 & 1.5129 & $\mathbf{C 3}$ & 5.7903 & 0.9245 \\
\hline $\mathbf{A 4}$ & 13.8749 & 1.4184 & $\mathbf{B 4}$ & 15.2365 & 1.4554 & $\mathbf{C 4}$ & 8.5707 & 0.9591 \\
\hline $\mathbf{A 5}$ & 34.8844 & 1.4632 & $\mathbf{B 5}$ & 3.6506 & 1.4993 & $\mathbf{C 5}$ & 19.7314 & 0.7273 \\
\hline $\mathbf{A 6}$ & 19.9011 & 1.3598 & $\mathbf{B 6}$ & 12.7664 & 1.4645 & $\mathbf{C 6}$ & 18.6195 & 0.8057 \\
\hline $\mathbf{A 7}$ & 28.4760 & 1.5645 & $\mathbf{B 7}$ & 34.8341 & 1.6207 & $\mathbf{C 7}$ & 33.4746 & 0.7359 \\
\hline $\mathbf{A 8}$ & 4.9958 & 1.3509 & $\mathbf{B 8}$ & 23.3576 & 1.4020 & $\mathbf{C 8}$ & 7.4120 & 0.7539 \\
\hline $\mathbf{A 9}$ & 6.0656 & 1.1797 & $\mathbf{B 9}$ & 9.1413 & 1.4358 & $\mathbf{C 9}$ & 16.1467 & 0.7533 \\
\hline $\mathbf{A 1 0}$ & 28.3728 & 1.4095 & $\mathbf{B 1 0}$ & 11.0645 & 1.3314 & $\mathbf{C 1 0}$ & 13.8137 & 0.7247 \\
\hline $\mathbf{A 1 1}$ & 10.3133 & 1.2199 & $\mathbf{B 1 1}$ & 6.6685 & 1.5384 & $\mathbf{C 1 1}$ & 20.6827 & 0.7585 \\
\hline $\mathbf{A 1 2}$ & 3.8465 & 1.2881 & $\mathbf{B 1 2}$ & 3.3515 & 1.4373 & $\mathbf{C 1 2}$ & 17.0459 & 0.7360 \\
\hline $\mathbf{A 1 3}$ & 21.8130 & 1.3464 & $\mathbf{B 1 3}$ & 11.8048 & 1.5220 & $\mathbf{C 1 3}$ & 9.9712 & 0.9400 \\
\hline $\mathbf{A 1 4}$ & 14.1379 & 1.3383 & $\mathbf{B 1 4}$ & 30.6947 & 1.7319 & $\mathbf{C 1 4}$ & 22.2235 & 0.8487 \\
\hline
\end{tabular}

Pred. : Prediction ; Dev. : Deviation

\section{Conclusions}

Quantitative analysis of food adulterants is an important for health, wealth and economic issue that needs to be fast and simple. In the light of the statistical results, it can be concluded that FT-MIR spectroscopy using ATR accessory coupled with chemometric tools can be used to quantify the adulteration of sesame oil with colza, soybean and sunflower oils. The level of adulterants (0-35\%) was successfully determined by PLSR models. The potential adulterants were detected in sesame oil at the level low than $3 \%$ detection limit in their binary mixtures (CO-SO: 1.47\%; SBO-SO: 2.67\%; SFO-SO: $2.34 \%$ ).

This approach represents a facile and convenient means for monitoring sesame oil quality with the advantage of ease of operation, high sample turnover and any sample preparation step.

\section{References}

[1] Namiki, M. Antioxidants/antimutagens in food.Critical Reviews of Food Science and Nutrition, 29, 1990, 273-300

[2] Hirata, F., Fujita, K., Ishikura, Y., Hosoda, K. Hypocholesterolemic effect of sesame lignan in humans. Atherosclerosis, 22, $1996,135-136$

[3] Kita, S., Matsumura, Y., Tanida, Y., Morimoto, S. Antihypertension effects of sesamin. Biological and pharmaceutical Bullet, 21, 1998, 469-73.

[4] Lee, J., Kim, M., Choe, E. Study on the changes of tocopherols and lignans and the oxidative properties of roasted sesame oil during manufacturing andstorage. Korean Journal of Food Science and Technology 40, 2008, 15-20.

[5] Abou-Gharbia, H. A., Shehata, A. A., \& Shahidi, F. Effect of processing on oxidative stability and lipid classes of sesame oil.Food Research International, 33, 2000, 331-340.

[6] Tay, A., Singh, R. K., Krishnan, S. S., \& Gore, J. P. Authentication of olive oil adulterated with vegetable oils using Fourier transforms infrared spectroscopy.LWT-Food Science and Technology, 35, 2002, 99-103

[7] John H. Kalivas, Constantinos A. Georgiou, Marianna Moira, Ilias Tsafaras, Eleftherios A. Petrakis, George A. Mousdis. Food adulteration analysis without laboratory prepared or determined reference food adulterant values. Food Chemistry 148, 2014, 289293

[8] Gharibzahedi, S. M. T., Mousavi, S. M., Hamedi, M., \&Khodaiyan, F. Determination and characterization of kernel biochemical composition and functional compounds of Persian walnut oil. Journal of Food Science and Technology, 51, $2014,34-42$.

[9] Harwood, J., \&Aparicio, R. Handbook of olive oil, analysis and Properties. Maryland: Aspen Publishers. 2000.

[10] Oliveira, R., Rodrigues, M. F., \& Bernardo-Gil, M. G. Characterization and supercritical carbon dioxide extraction of walnut oil.Journal of the American Oil Chemists Society, 79, 2002, 225-230.

[11] Christopoulou, E., Lazarakia, M., Komaitis, M., \& Kaselimis, K. Effectiveness of determinations of fatty acids and triglycerides for the detection of adulteration of olive oils with vegetable oils. Food Chemistry, 84, 2004, 463-474.

[12] EI-Hamdy, A. H., \& El Fizga, N. K. Detection of olive oil adulteration by measuring its authenticity factor using reversed-phase high-performance liquid chromatography. Journal of Chromatography A, 708, 1995, 351-355.

[13] Fasciotti, M., \& Nettoa, A. D. P. Optimization and application of methods of triacylglycerol evaluation for characterization of olive oil adulteration by soybean oil with HPLC-APCI-MS-MS. Talanta, 81, 2010, 1116-1125.

[14] Zabaras, D., \& Gordon, M. H. Detection of pressed hazelnut oil in virgin olive oil by analysis of polar components: Improvement and validation of the method. Food Chemistry, 84, 2004, 475-483.

[15] Rezanka, T., \& Rezankova, H. Characterization of fatty acids and triacylglycerols in vegetable oils by gas chromatography and statistical analysis. Analytica Chimica Acta, 398, 1999, 253-261.

[16] Hilali, M., Charrouf, Z., Soulhi, A., Hachimi, L., \& Guillaume, D. Detection of argan oil adulteration using quantitative campesterol GC-analysis. Journal of the American Oil Chemists Society, 84, 2007, 761-764.

[17] Rachid Salghi, Wolfgang Armbruster, Wolfgang Schwack. Detection of argan oil adulteration with vegetable oils by highperformance liquid chromatography-evaporative light scattering detection. Food Chemistry 153, 2014, 387-392.

[18] Gonzálvez, A., Armenta, S., \& de la Guardia, M. Adulteration detection of argan oil by inductively coupled plasma optical emission spectrometry. Food Chemistry, 121, 2010, 878-886.

[19] Hai Zheng \& Wang Jun. Electronic nose and data analysis for detection of maize oil adulteration in sesame oil. Sensors and Actuators B, 119, 2006, 449-455.

[20] Peng Dan, Bi Yanlan, Ren Xiaona, Yang Guolong, Sun Shangde, Wang Xuede. Detection and quantification of adulteration of sesame oils with vegetable oils using gas chromatography and multivariate data analysis. Food Chemistry, 188, 2015, 415-421.

[21] Lee Wei-Ju, Su Nan-Wei, Lee Min-Hsiung, Lin Jui-Tsung. Assessment of authenticity of sesame oil by modified Villavecchia Test and HPLC-ELSD analysis of triacylglycerol profile. Food Research International, 53, 2013, 195-202.

[22] Zhang Liangxiao, Shuai Qian, Li Peiwu, Zhang Qi, Ma Fei, Zhang Wen, Ding Xiaoxia. Ion mobility spectrometry fingerprints: A rapid detection technology for adulteration of sesame oil. Food Chemistry, 192, 2016, 60-66.

[23] Guillen, M. D., \& Cabo, N. Some of the most significant changes in the Fouriertransform infrared spectra of edible oils under oxidative conditions.Journal of the Science of Food and Agriculture, 80, 2000, 2028-2036. 
[24] Lerma-García, M. J., Ramis-Ramos, G., Herrero-Martínez, J. M., \&Simó-Alfonso, E. F. Authentication of extra virgin olive oils by Fourier-transform infraredspectroscopy.Food Chemistry, 118, 2009, 78-83.

[25] Oussama, A., Elabadi, F., Platikanov, S., Kzaiber, F., Tauler, R. Detection of Olive Oil Adulteration Using FT-IR Spectroscopy and PLS with Variable Importance of Projection (VIP) Scores. J Am Oil Chem Soc, 89, 2012, 1807-1812.

[26] Roggo, Y., Chalus, P., Maurer, L., Lema-Martinez, C., Edmond, A., \& Jent, N. Areview of near infrared spectroscopy and chemometrics in pharmaceutical technologies.Journal of Pharmaceutical and Biomedical Analysis, 44, 2007, 683-700.

[27] Savitzky, A. \& Golay, M.J.E. Smoothing and differentiation of data by simplified least-squares procedures. Analytical Chemistry, 36, 1964, 1627-1639.

[28] Norris, K.H. \& Williams, P.C. Optimization of mathematical treatments of raw near infrared signal in the measurement of protein in hard Red Spring wheat, I: influence of particle size. Cereal Chemistry, 62, 1984,158-165.

[29] [29] Iñón, F. A., Garrigues, J. M., Garrigues, S., Molina, A., and de la Guardia, M. Selection of calibration set samples in determination of olive oil acidity by partial least squares-attenuated total reflectance-Fourier transform infrared spectroscopy. Analytica Chimica Acta, 489, 2003, 59-75.

[30] Gurdeniz, G., Tokatli, F., \& Ozen, B. Differentiation of mixtures of monovarietal olive oils by mid-infrared spectroscopy and chemometrics. European Journal of Lipid Science and Technology, 109, 2007, 1194-1202.

[31] Wold, S., Esbensen, K., Geladi, P. Chemom. Intell. Lab. Syst. 2, 1987, 37.

[32] Vandeginste, B.G.M., Massart, D.L., Buydens, L.M.C., Jong, S.D.E., Lewi, P.J., Smeyers-Verbeke, J. Handbook of Chemometrics and Qualimetrics: Part B, Elsevier, Amsterdam, The Netherlands, 1998, 88.

[33] Brown, S.D., Tauler, R., Walczak, B. Comprehensive Chemometrics: Chemical and Biochemical Data Analysis. 2009 , vol. 2. Elsevier, Amsterdam.

[34] Martens, H. \& Naes, T. Multivariate calibration. Chichester: Wiley. 1989.

[35] Mohammadreza Khanmohammadi, Amir Bagheri Garmarudi, Keyvan Ghasemi, Salvador Garrigues, Miguel de la Guardia. Artificial neural network for quantitative determination of total protein in yogurt by infrared spectrometry. Microchemical Journal 91, 2009, 47-52.

[36] Geladi, P. \& Kowalski, B. Partial least-squares regression: a tutorial. Anal Chim Acta 185, 1986, 1-17.

[37] Guillen, M. D., \& Cabo, N. Characterization of edible oils and lard by Fourier transform infrared spectroscopy. Relationships between composition and frequency of concrete bands in the fingerprint region. Journal of the American Oil Chemists Society, 74, 1997, 1281-1286

[38] Rohman, A., \& Che Man, Y.B. Food Res. Int., 43, 2010, 886-892.

[39] Lopez-Sanchez, M., Ayora-Canada, M.J., Molina-Diaz, A. J. Agric. Food Chem., 58, 2010, 82-87.

[40] Yap, K. Y.-L., Chan, S. Y., \& Lim, C. S. Infrared-based protocol for the identification and categorisation of ginseng and its products. Food Research International, 40, 2007, 643-652.

[41] Anklam, E., \& Bantaglia, R. Food analysis and consumer protection. Trends in Food Science and Technology, 12, 2001, 71-102.

[42] Christy, A. A., Kasemsumran, S., Du, Y., \& Ozaki, K. The detection and quantification of adulteration in olive oil by near-infrared spectroscopy and chemometrics. Analytical Sciences, 20, 2004, 935-940.

[43] Pirie, A.; Singh, B. \& Islam, K. Ultra-violet, visible, near-infrared, and mid-infrared diffuse reflectance spectroscopic techniques to predict several soil properties. Aust J Soil Res, 43, 2005, 713-721.

[44] Rohman, A., \& Che Man, Y.B. The use of Fourier transform mid infrared (FT-MIR) spectroscopy for detection and quantification of adulteration in virgin coconut oil. Food Chemistry 129, 2011, 583-588

[45] Durante, C., Cocchi, M., Grandi, M., Marchetti, A., \& Bro, R. Application of N-PLS to gas chromatographic and sensory data of traditional balsamic vinegars of Modena. Chemometrics and Intelligent Laboratory Systems, 83(1), 2006, 54-65.

[46] Rezaei, B., Khayamian, T., \& Mokhtari, A. Simultaneous determination of codeine and noscapine by flow-injection chemiluminescence method using N-PLS regression. Journal of pharmaceutical and biomedical analysis, 49(2), 2009, 234-239. 